\title{
Subdural empyema as a complication of acute otitis media in a 67-year-old patient
}

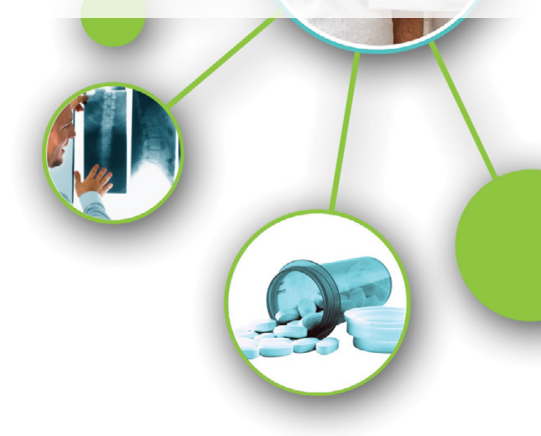

The acute otitis media can be caused by viruses (nonsuppurative inflammation) and bacteria (suppurative inflammation). The suppurative inflammation is a risk factor of endocranial complications, including subdural empyema burdened with the high mortality. The authors present a clinical case of 67 -year-old man treated by surgery because of subdural empyema in the course of the acute suppurative otitis media.

\section{Keywords: acute otitis media, subdural empyema}

\section{Introduction}

Otitis media is a common disease. In its pathophysiology, the auditory trumpet plays an important role, the task of which is, i.e. an effective limiting the passage of microorganisms from the nasopharynx to the tympanic cavity. The increased risk of otitis media causes all the mechanical and functional factors that impair the obstruction of the auditory tube, such as hypertrophic tonsils or mucociliary clearance disorders. Due to the etiology, the image in the otoscopic examination and the course of infection distinguish three types of otitis media: acute, chronic and exudative $(1,2)$. Acute otitis media may have both a viral etiology (inflammation: RSV - respiratory syncytial virus, rhinovirus, coronavirus, adenovirus, CMV - Cytomegalovirus, influenza and parainfluenza virus) as well as bacterial infection (inflammation) [1-3].

Acute purulent otitis media causes such pathogens as Streptococcus pneumoniae (most commonly, about 40\%), Haemophilus influenzae, Moraxella catarrhalis and S. viridans, Staphylococcus aureus or Gram-negative rods $[1,4,5]$. Nasopharyngeal continuity is the most common route of infection, and the major ailments include nocturnal otalgia, impaired hearing, chills, fever, asthenia and malaise [6,7].

The complication of acute purulent otitis media may take the form of soft tissues and bones infiltration, and penetration into the structures located inside the skull - this may then take the form of a limited purulent foci in the brain tissue or an out-of-focus or subdural foci. Middle ear inflammation, including acute purulent otitis media, accounts for almost $1 / 3$ of the risk factors for subdural empyema [8-10].

\section{Case report}

A 67-year old patient was admitted urgently to the Department of Otolaryngology and Laryngologic Oncology due to acute purulent otitis media of the left ear with associated temperature up to $39^{\circ} \mathrm{C}$. On the admission the patient underwent a HRCT (high resolution computed tomography) of a temporal bone, on which shading of mastoid cells (with fluid levels) on the left side, and nonspecific pathological soft tissue masses filling the left mastoid antrum, hypo- and mesotympanum, surrounding the ossicles and occupying sinus of the eardrum and the recess of nerve VII. The patient has not been treated otolaryngologically so far. Blood leukocytosis in the blood count (WBC - white blood cells: $\left.19.52 \times 10^{3} / \mu \mathrm{l}\right)$. Two seizures occurred during hospitalization, which resolved with diazepam. The patient underwent paracentesis on the left side, resulting in an outflow of purulent content. The next day after admission, at night, the patient was urgently consulted neurologically and internistically due to a sudden deterioration of the general condition. After neurological consultation, the CNS (central nervous system) CT (computed tomography) was recommended, in which no pathological areas were found. After another

\section{Maciej Śniegocki ${ }^{1}$, Agnieszka Nowacka*1, Dawid Lewko ${ }^{3}$, Wojciech Smuczyński², Kamila Woźniak ${ }^{1}$, Wojciech Kaźmierczak4, Katarzyna Pawlak- Osińska ${ }^{5}$}

${ }^{1}$ Department of Neurosurgery, Neurotraumatology and Paediatric Neurosurgery, University in Toruń

${ }^{2}$ Department of Neurotraumatology, University in Toruń

${ }^{3}$ Department of Clinical Chemistry and Biochemistry, Medical University in Łódź

${ }^{4}$ Department of Otolaryngology and Oncology, University in Torun

${ }^{5}$ Division of Pathophysiology of Hearing and Balance Systems, University in Toruń

*Author for correspondence:

dr.agnieszka.nowacka@gmail.com 
convulsive seizure, the patient required intubation and was transfer to the Anesthesiology and Intensive Care Clinic. Upon admission, the patient was in a severe general condition, unconscious, with symptoms of neuroinfection, requiring mechanical ventilation. The cerebrospinal fluid was collected - features of bacterial infection in general examination. On the first day at Anesthesiology and Intensive Care Clinic, after laryngological consultation, a mastoidectomy has been performed, in which no purulent secretion was found. Wide-spectrum antibiotic therapy and multidirectional supportive treatment were applicated, resulting in gradual lowering of inflammation parameters and improvement of the general condition. On day 6, CNS CT was performed and the presence of a pathological fluid space with the mass effect was demonstrated. The consulting neurosurgeon qualified the patient for surgery. Intraoperatively, the presence of subdural empyema was found, which was drained. On the following days, the patient's condition gradually improved. White blood cell counts decreased to normal values (WBC: $8.97 \times 10^{3} / \mu \mathrm{l}$ ). Mechanical ventilation was stopped. A CNS CT scan was performed and did not show new pathological fluid spaces. On the 11th day the patient was extubated, and a day later respiratory and haemodynamically stable was transferred to the Department of Neurosurgery, Neurotraumatology and Pediatric Neurosurgery. Until the end of the stay, the patient did not get feverish, and at the time of discharge did not have any neurological deficits.

\section{Discussion}

Infection from the middle ear can spread along the branches of the blood and lymphatic vessels, nerves, and also through congenital bone defects or those acquired as a result of injury or inflammation [11-13]. In the discussed case, the HRCT examination confirmed several small bone defects on the left side in tegmen tympani and tegmen mastoideum (more pronounced than on the right side), which most probably supports the coexistence of bone destruction and dehiscence. In addition, in the vicinity of the described bone defects, small fine air bubbles were also visible, which as a result of bone and dura disruption passed from the middle left ear to the cranial cavity. The presence of air bubbles was also found in the middle fossa of the skull, forward of the pyramid, along the temporal bone. The radiological image confirmed that due to the interruption of the bone barrier, the inflammatory process spread to the inside of the skull.

In the case of subdural empyema, the early stage of the disease may give false negative results in computed tomography. Therefore, in order to make an early accurate clinical diagnosis, it is important to supplement the diagnosis with contrast-enhanced magnetic resonance imaging [11-13].

In presented case, after urgent neurological and internist consultations, CNS CT with contrast was performed, in which the presence of pathological areas were not demonstrated. However, after a few days, the re-examination revealed the presence of a pathological fluid space with the displacement of brain structures, which allowed changing the therapy path and qualify the patient for surgery, during which the subdural abscess was found. The presented case confirms that in the case of clinical doubts, imaging should be repeated.

Only half of patients with subdural empyema successfully undergo convalescence, while others experience permanent neurological deficits or death [14-16]. The pharmacological and surgical treatment applied in this patient resulted in significant improvement in general condition and protected against neurological deficits.

\section{Conclusion}

1. Subdural empyema is with one complication of acute purulent otitis media.

2. In case of any clinical doubts, it seems appropriate to repeat the imaging examinations.

3. In doubtful cases in patients with acute purulent otitis media, it seems advisable to extend their diagnosis with contrast-enhanced magnetic resonance imaging. 


\section{REFERENCES}

Kuthan R. Treatment of acute otitis media in the light of the latest guidelines. Drug Poland. 24, 57-69 (2014).

Thomas JP, Berner R, Zahnert T. Acute otitis media - a structures approach. Dtsch Arztebl Int. 111, 151-159 (2014).

Kuczkowski J. Current problems in the diagnosis and treatment of acute and exudative otitis media. Forum Medycyny Rodzinnej 5, 287-294 (2011).

Mittal R, Kodiyan J, Gerring R, et al. Role of innate immunity in the pathogenesis of otitis media. Int. J. Infect. Dis. 29, 259-267 (2014).

Maranhão A, Andrade J, Godofredo V, Matos R, Penido N. Epidemiology of intratemporal complications of otitis media. Int. Arch. Otorhinolaryngol. 18, 178-183 (2014).
Wanna GB, Dharamsi LM, Moss JR, Bennett ML, Thompson RC, Haynes DS. Contemporary management of intracranial complications of otitis media. Otol. Neurotol. 31, 111-117 (2010).

Qureishi A, Lee Y, Belfield K, et al. Update on otitis media - prevention and treatment. Infect. Drug Resist 7, 15-24 (2014).

Vergison A. Microbiology of otitis media: A moving target. Vaccine 26 Suppl 7, G5-G10 (2008).

Klein JO. Is acute otitis media a treatable disease? N. Engl. J. Med. 13, 168169 (2011).

Qirjazi B, Bardhyli D, Hoxhallari X. Multiple Complications Due to Subacute Suppurative Otitis Media. Balkan Med. J. 29, 203-204 (2012).

Greenlee JE. Subdural empyema. Curr. Treat Options Neurol. 5, 13-22 (2003).
Mostafa BE, El Fiky LM, ElSharnouby MM. Complications of suppurative otitis media: still a problem in the 21 st century. J. Otorhinolaryngol. Relat. Spec. 71, 87-92 (2009).

Wetmore RF. Complications of otitis media. Pediatr. Ann. 29, 637-646 (2000).

Ibrahim SI, Cheang PP, Nunez DA. Incidence of meningitis secondary to suppurative otitis media in adults. J. Laryngol. Otol. 124, 1158-1161 (2010).

Osborn MK, Steinberg JP. Subdural empyema and othersuppurative complications of paranasal sinusitis. Lancet Infect. Dis. 7, 62-67 (2007).

Penido NO, Borin A, Iha LCN, et al. Intracranial complications of otitis media: 15 years of experience in 33 patients. Otolaryngol. Head Neck Surg. 132, 37-42 (2005). 